\title{
Simulation of low velocity impact on composite hemispherical shell
}

\begin{abstract}
Impact simulation with finite element analysis is an appropriate manner to reduce the cost and time taken to carry out an experimental testing on a component. In this study, the impact behavior of the composite hemispherical shell induced by low velocity impact is simulated in ABAQUS software with finite element method. To predict the responses of Kevlar fabric/polyester, glass fabric/polyester and carbon fabric/polyester in the form of a hemisphere, once as one layer and then as a three-layered composite under applied force by an anvil. The sequences of layers are changed, to investigate and compare the occurred alternations in the amount of energy absorption, impact force and specific energy absorption (SEA). The comparison of results showed that the highest and the lowest quantity of energy absorption and SEA belong to Carbon/Glass/Kevlar (CGK) and Kevlar/Carbon/Glass (KCG) respectively.
\end{abstract}

Keyword: Energy absorption; Finite element modeling; Impact behavior; Shell structure 